\title{
Formulation of Stray Loss in Medium Power Inverter-fed Induction Motors
}

\author{
Rajendra Kumar, Bikash Sah, Member, IEEE, Praveen Kumar, Senior Member, IEEE
}

\begin{abstract}
The work presents a fast and accurate empirical stray loss model for inverter fed three-phase induction motors (IMs). The model is intended to quantify the SL both at the design stage and, normal operation of the IM. The development of the model considers the variations of frequency, switching frequency and motor's loading. Test data of 128 operating points of two training IMs are used to develop the model. The accuracy and prediction capability of the proposed model is presented with the measured SL values for 128 operating points of another set of two IMs of the same specifications and different geometries.
\end{abstract}

Index Terms-Induction motor, core loss, stray loss, motor efficiency, inverter, pulse-width modulation (PWM) supply.

\section{INTRODUCTION}

G Rowing concern of energy saving has made motor efficiency a prime necessity among design engineers and motor-driven systems manufacturers. An accurate measure of motor efficiency is always required to operate an existing motor in the high-efficiency region and to design an energyefficient motor [1]. The efficiency estimation uses two terms evaluated precisely viz. motor's output power and the sum of all the losses incurred. The motor's output power can be measured directly with high precision [2], whereas the losses in the motor, especially the magnetic losses, have always been challenging. These magnetic losses include core losses (CL) and stray load losses (SL). Both these losses depend upon the time and space distribution of flux density in a motor. If the motor is supplied power with a PWM inverter, these magnetic losses become more prominent and complex to evaluate due to higher-order harmonics. The evaluation of CL for an inverter fed motor is widely researched and, there exist several models of CL (from modified Steinmetz equation [3] to two or three terms CL model with variable loss coefficients and, hybrid CL models [4]. On the other hand, the SL still lacks a straightforward and precise model representation for inverter fed motors.

SL in an induction motor (IM) is the additional CL, attributed to the various space and time harmonics of magnetic flux density [5], [6]. The space-harmonics results from the placement of coils in the slotted stator and variation of fluxpath permeance [7]. In contrast, the time-harmonics are linked to the non-linearity (in MMF-flux linkage) introduced by the magnetic saturation and the inverter voltage harmonics. The impact of time harmonics depends upon the ratio of fundamental to the switching frequency [8]. This ratio varies from 20 (medium power application) to 200 (small power applications) in the majority of inverter fed IM-drives [9], [10].

When addressing the SL, literature is broadly restricted to the space harmonics and sinusoidal excitation [7], [11], [12]. [7] and [11], present theoretical expressions to estimate the SL in a grid-connected IM. These expressions are rather complex, require the motor's complete geometry details, and need modifications as the slots' geometry changes. In a lumped element-based approach, Alger et al. present an extended equivalent circuit of IM to include the impact of harmonics [11]. Direct and exact evaluation of these coupled lumped parameters is strenuous.

Evaluation of SL in the post-process of FEA results is another approach used in recent works [13], [14]. However, unlike CL, which has three constituents, SL consists of multiple sources, making the post-process estimation complex in all the aspects-time, data storage, and computation [15].

In the attempt to redress these evaluation intricacies, [12], [16], [17] present different SL empirical models. The models presented in these works are developed with a logical hypothesis supported by the statistical analysis on a large set of measured SL data. Such models accelerate the iterative motor design process and require less response time when incorporated in motor control than purely physics-based methods. However, all the available SL models [12], [16], [17] are exclusively developed for a sinusoidal power supply. The SL models for PWM excited IMs are not addressed extensively and, literature still lacks a simple and generalised formulation of SL in inverter fed IM.

The work presented in this paper aimed to develop a simple and accurate SL model for an inverter fed IM, which:

- can be used in the design phase to minimise the SL.

- applies to the motors already in operation, whose detailed design and material data are not available except for the name-plate data.

- can distinguish the IMs of the same power rating w.r.t. their SL values. For example, two motors of the same power ratings but different efficiency classes will have different SL values, and the model should predict it.

- be applied to determine SL for different loading of the motors.

The model presented in this work uses IM's equivalent circuit parameters, output power, current, current's total harmonic distortion as input variables. All these input variables are available at both the design and testing stages, making the model capable of fulfilling the four criteria mentioned above. 
The organisation of the paper is as follows: Section II reports the theoretical framework of the SL in an IF-IM.Section III presents SL measurements and loss segregation. Proposed SL formulation and its estimation accuracy is detailed in Section IV. Section V concludes the paper.

\section{BACKGROUND: StRAY LOSSES IN INVERTER FED IM}

Primary sources of SL in an IM are the various leakage fluxes, space and, time harmonics of the magnetic flux density $(B(t))$. These space and time harmonics of $B(t)$ are traced to the variation of magnetomotive force MMF. This section describes the space and harmonics in an inverter fed IM and their correlation with SL.

\section{A. Space harmonics with sinusoidal excitation}

Fourier decomposition of the magnetomotive force (MMF) generated by a distributed winding of one-phase (in a threephase machine) is given as [11]:

$$
F_{A}(\theta, t)=\frac{2}{\pi} N_{S} i(t) \sum_{v=1}^{n} \frac{K_{W}(v)}{v} \sin (v p \theta)
$$

where,

$$
i(t)=I_{m} \sin (\omega t)
$$

, $K_{W}(v)$ is the winding factor of the $v$-th space harmonic. $N_{S}$ and, $p$ are, respectively, per phase number of turns in stator winding and, pole-pairs. For a three-phase machine drawing a balanced set of currents, the net MMF is given as the sum of MMFs of all the three phases, which are 120 shifted in space and it is:

$$
F_{S}(\theta, t)=F_{A}(\theta, t)+F_{B}(\theta, t)+F_{C}(\theta, t)
$$

Using (1) and (2), the net MMF is given as:

$$
F_{S}(\theta, t)=\frac{3}{2}\left[\begin{array}{l}
A_{1} \cos (\omega t-\theta)-\frac{A_{5}}{5} \cos (\omega t+5 \theta)+ \\
\frac{A_{7}}{7} \cos (\omega t-7 \theta) \ldots \ldots
\end{array}\right]
$$

where,

$$
A_{v}=\frac{2}{\pi} N_{S} I_{m} \cdot K_{W}(v)
$$

Expression (3) represents multiple travelling waves rotating in different directions. All these MMF harmonic waves travel with a speed less than the synchronous speed i.e., the speed of the fundamental MMF-wave.The MMF waves corresponding to space harmonic number $v=6 k-1$ rotate in the reverse direction of the MMF waves of fundamental and, $6 k+1$ harmonic numbers. The space harmonics of order higher than 7 are negligible [6] and hence are neglected.

\section{B. Space harmonics with inverter fed excitation}

For an inverter-fed IM, the motor currents are expressed as:

$$
\begin{gathered}
i_{a}(t)=\sum_{n=1}^{h} I_{m}(n) \sin (n \omega t+\phi(n)) \\
i_{b}(t)=\sum_{n=1}^{h} I_{m}(n) \sin \left(n \omega t+\phi(n)-\frac{2 \pi}{3}\right)
\end{gathered}
$$

$$
i_{c}(t)=\sum_{n=1}^{h} I_{m}(n) \sin \left(n \omega t+\phi(n)+\frac{2 \pi}{3}\right)
$$

Evaluation of (1)-(3) for the MMF in relation to the current expressions (5)-(6) gives:

$$
F_{S}(\theta, t)=F_{1}(\theta, t)+F_{5}(\theta, t)+F_{7}(\theta, t)+\ldots
$$

where,

$$
\begin{gathered}
F_{1}(\theta, t)=\sum_{v=1}^{n} A_{v} \cos (v \omega t-p \theta) \\
F_{5}(\theta, t)=\sum_{v=1}^{n} A_{v} \cos (v \omega t+5 p \theta) \\
F_{7}(\theta, t)=\sum_{v=1}^{n} A_{v} \cos (v \omega t-7 p \theta)
\end{gathered}
$$

From expressions (10)-(12), it is seen that the harmonics in current superimpose various high-frequency waves to the MMF waves corresponding to the fundamental frequency. All these rotating MMF waves produce additional SL.

\section{Correlation of SL with the inverter power supply}

The major components of SL viz. surface core loss, tooth flux pulsation loss and, phase belt pulsation loss are the additional eddy current losses in reaction to the space harmonics of the $B(t)$. The expressions of these losses derived using the diffusion equation and Faraday's law show that these losses vary directly as the square of MMF [6], [7]. Hence, SL in a cumulative effect of all the MMF harmonics for an inverter fed motor can be expressed as:

$$
\begin{gathered}
S L=\sum_{v=1}^{n} S L(i)=\sum_{v=1}^{n} K_{i} F_{i}^{2} \\
\Rightarrow S L=K_{1} F_{1}^{2}\left(1+k_{2}\left(\frac{F_{2}}{F_{1}}\right)^{2}+k_{3}\left(\frac{F_{3}}{F_{1}}\right)^{2}+\ldots\right)
\end{gathered}
$$

where, $S L(i)$ is the net SL with $i$-th time-harmonic and all the space harmonics. $F_{i}$ is the magnitude of the MMF corresponding to the i-th current harmonic (as shown by (7)(9)). $K_{i}$ is the proportional coefficient of the SL-MMF relation. With a linear relation of MMF with current, the series sum used in (11) is directly proportional to the total harmonic distortion (THD) in the motor-current (a directly measurable quantity), and hence the SL in an inverter fed motor can be written as

$$
S L=K_{1} F_{1}^{2} f(T H D)
$$

where, $f(T H D)$ is a single valued monotonic function of motor's current THD. The determination of this function is explained in section-IV, which uses the experimental results of SL. The test procedure and the experimental results are detailed in the next section, i.e. section-III.

\section{EXPERIMENTAL Estimation OF SL}

Total four squirrel cage induction motors of the same power rating but different slot geometries are selected to record the 
TABLE III

variation of SL with inverter power supply. Nameplate details of these motors are given in Table-1 and, Table-III shows their slot geometries. Two prominent sources of the SL i.e., toothsaturation and permeance variation are very specific to the slot shape. The major limitations of existing analytical SL methods [7], [11], [14] is that, they are developed for the basic geometries of the slots and, hence it is not possible with these expressions to differentiate two IMs of same number of slots but different slot geometries (except the slot-opening) for SL. The purpose of selection of these motors is, hence, to examine the SL variation among the IMs of the same power rating and, pole pairs but different slot geometries.

TABLE I

SPECIFICATIONS OF TESTED IMS

\begin{tabular}{|c|c|c|c|c|}
\hline \multicolumn{2}{|c|}{$\begin{array}{c}\text { Line voltage/Frequency: } 200 \mathrm{~V} / 50 \mathrm{~Hz} \\
\text { Stator slots: } 36\end{array}$} & \multicolumn{2}{|c|}{$\begin{array}{l}\text { Pole-pairs }(p): 2 \\
\text { Rotor slots: } 28\end{array}$} & $P_{\text {Out }}(\mathrm{kW}): 2.5$ \\
\hline \multirow[t]{2}{*}{ Motor } & \multicolumn{2}{|c|}{ Design } & \multirow{2}{*}{$\begin{array}{l}\text { Rated Speed } \\
\text { (rpm) }\end{array}$} & \multirow{2}{*}{$\begin{array}{l}\text { Cage bar } \\
\text { material }\end{array}$} \\
\hline & Stator & Rotor & & \\
\hline M-1 & SD-1 & RD-1 & 1482 & Copper \\
\hline M-2 & SD-2 & RD-1 & 1482 & Copper \\
\hline M-3 & SD-2 & RD-2 & 1482 & Copper \\
\hline M-4 & SD-1 & RD-2 & 1482 & Copper \\
\hline
\end{tabular}

\section{A. Test operating points}

The ratio of switching frequency to the fundamental frequency varies highly among the various applications (from 10 to 200). Therefore, the IMs selected in this work (as shown Table I) are tested for a wide range of this ratio. Another important parameter affecting the SL is the fundamental frequency and loading of the motor. The SL measurements are performed to analyse all these parameters' impact on all the operating points as given in Table II.

TABLE II

IMS OPERATION DOMAIN

\begin{tabular}{llll}
\hline \hline & $f_{0}$ & Torque & $f_{0} / f_{\text {sw }}$ \\
& $(\mathrm{Hz})$ & $(\mathrm{Nm})$ & Ratio \\
\hline Minimum & 30 & 0 & 10 \\
Maximum & 60 & 14 & 200 \\
Steps & 4 & 5 & 5 \\
\hline \hline
\end{tabular}

\section{B. Measurement procedure}

SL for these four IMs is evaluated using the input-output test. The test is reported to be the most accurate among all the SL evaluation methods [18]. Since the power ratings of the IMs are not very high and they can be loaded upto their rated power, the input-output test is preferred. If the IM is inverter fed, all the CL and SL related tests require two sets of measurements- a)- load and no-load test with PWM power supply b)-load and no-load tests with sinusoidal excitation. These tests are performed as follows:

1) Rated load test: The test IM is coupled with magnetic power brake as load and loaded for different torque values as shown in Table-II. Voltages, currents and shaft torque are recorded at the thermal equilibrium.
Slot Shapes Of The TWo Design Prototypes

\begin{tabular}{|c|c|c|c|}
\hline Stator slots: Initial design & $\begin{array}{l}\text { SD1 } \\
\text { Dim. (mm) } \\
B_{s 0}=2.4 \\
B_{s 1}=6.0 \\
B_{s 2}=8.5 \\
R_{s}=2.75 \\
H_{s 0}=1.0 \\
H_{s 1}=0.67 \\
H_{s 2}=18.33\end{array}$ & Stator slots: Modified design & $\begin{array}{l}\text { SD-2 } \\
\text { Dim. (mm) } \\
B_{s 0}=1.5 \\
B_{s 1}=6.0 \\
B_{s 2}=9.5 \\
R_{s}=2.5 \\
H_{s 0}=0 \\
H_{s 1}=1.67 \\
H_{s 2}=18.0\end{array}$ \\
\hline Rotor slots: Initial design & RD1 & Rotor slots: Modified design & RD-2 \\
\hline $\mathrm{B}_{s 1}$ & $\begin{array}{l}\text { Dim. (mm) } \\
B_{s 01}=0 \\
B_{s 0}=5.5 \\
B_{s 1}=6.32 \\
B_{s 2}=3.17 \\
H_{s 0}=0.3 \\
H_{s 1}=1.0 \\
H_{s 2}=2.5 \\
H_{s 3}=14.0\end{array}$ & 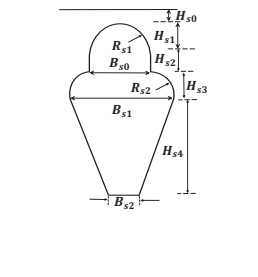 & $\begin{array}{l}\text { Dim. }(\mathrm{mm}) \\
B_{s 01}=0 \\
B_{s 0}=5.0 \\
B_{s 1}=6.32 \\
B_{s 2}=1.50 \\
H_{s 0}=0.3 \\
H_{s 1}=2.25 \\
H_{s 2}=1.25 \\
H_{s 3}=1.80 \\
H_{s 4}=14.0 \\
R_{1}=2.4 \\
R_{2}=3.12\end{array}$ \\
\hline
\end{tabular}

2) No-load test: The no-load (NL) test is performed with the hot motor after the load test. The friction and windage losses and core loss are obtained from the no-load test at different supply voltages with sinusoidal power supply. Fig. 1 (a)-(b) show the NL-test results. Detailed step-wise procedure for these test in line with IEC-60034-3-2 is discussed in [19],[20]. Given the fact that, SL-estimation is highly sensitive to the torque and current measurements [29], the equipments used are selected in compliance with IEEE 112-B (i.e, $0.2 \%$ accuracy for voltage and current measurements and, $0.5 \%$ accuracy for toque and speed measurements).

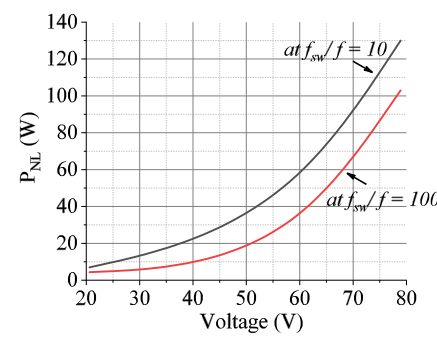

(a)

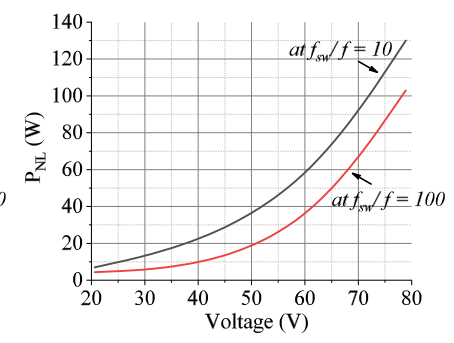

(b)
Fig. 1. No-load test results for at(a) M1, (b) M2.

The measurement set-up for these test (as shown in Fig.2 comprises: a).torque sensor (Kistler 4503B) and CoMo Torque Type 4700 data acquisition system. b) 2-level, H-bridge threephase inverter c). $1.5 \mathrm{~kW}$ IM motor prototype d). Digital oscilloscope: YOKOGAWA DLM2054 with voltage and current probes e). Voltage and current transducers: LEM sensors LV25-P and LA 15P f). dSpace DS1103 for data acquisition g).Photo-tachometer.

SL from these test data is obtained as difference of CL between the loaded and no-load conditions of the IM [21],[14] i.e.,

$$
S L=P_{\text {Core } \_} L-P_{\text {Core }} N L
$$

where, $P_{\text {Core_L }}$ and, $P_{\text {Core } N L}$ are, respectively, the $\mathrm{CL}$ at loaded and no-load conditions for the same voltage, $f$ and $f_{s w}$. The CL, here, is calculated using the cycle-averaged value of 

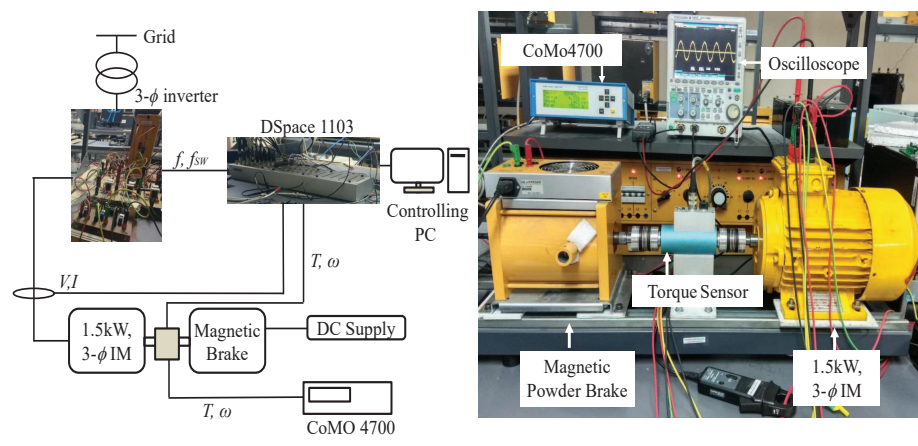

Fig. 2. Experimental setup: Schematic and test-bench

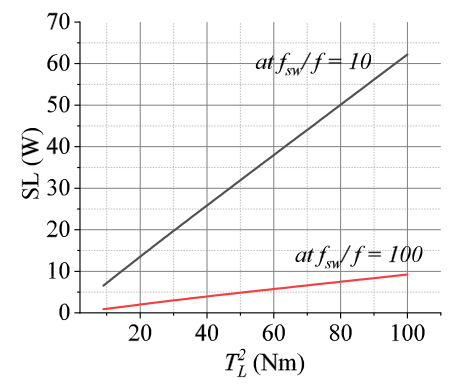

(a)

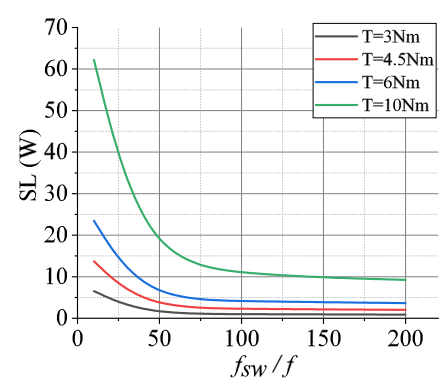

(c)

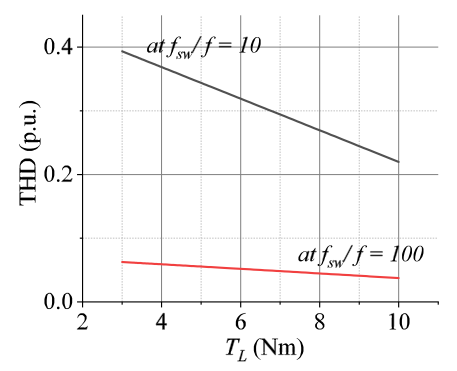

(e)

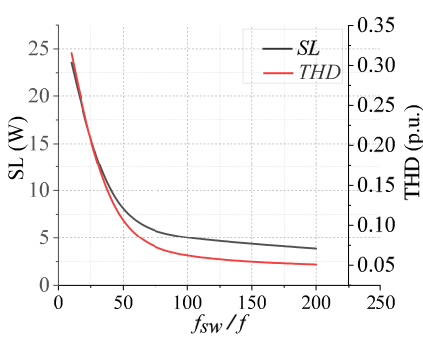

(b)

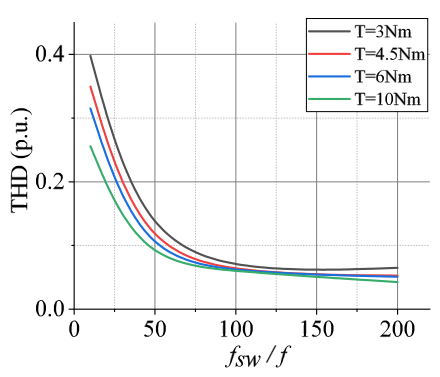

(d)

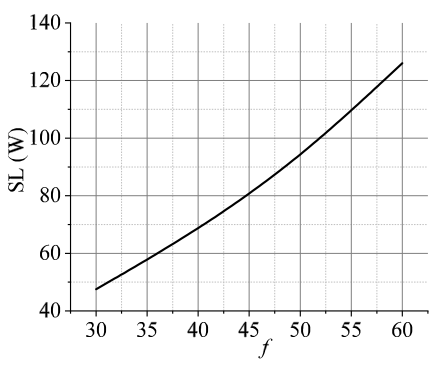

(f)
Fig. 3. Measurement results for IM-M1 (a) Variation of SL with $T_{L}^{2}$ for high and small values of $f_{S W} / f$ at, (b) Correlation of SL and THD variations with $f_{S W} / f$, (c) Variation of SL with $f_{S W} / f$ for different $T_{L}$, (d) Variation of THD with $f_{S W} / f$ for different $T_{L}$, (e) Impact of loading on THD at high and small $f_{S W} / f$, (f) SL variation with $f$ at constant $f_{S W} / f$ $(=10)$ and, $T_{L}(=10 \mathrm{Nm}) . f$, in all these plots, $=30 \mathrm{~Hz}$.

input power $\left(P_{I n}\right)$ and then, applying the input-output power balance as:

$$
P_{\text {Core }}=P_{\text {In }}-P_{C u S}-P_{C u R}-P_{\text {Out }}
$$

where, $P_{C u S}$ and, $P_{C u R}$ are the stator and, rotor copper losses, respectively, determined using the frequency and temperature dependant values of stator and rotor resistances (i.e., $R_{S}\left(T, f_{i}\right)$,

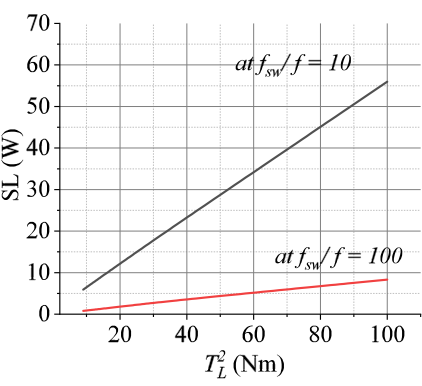

(a)

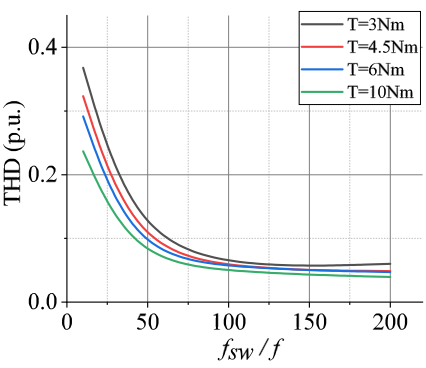

(c)

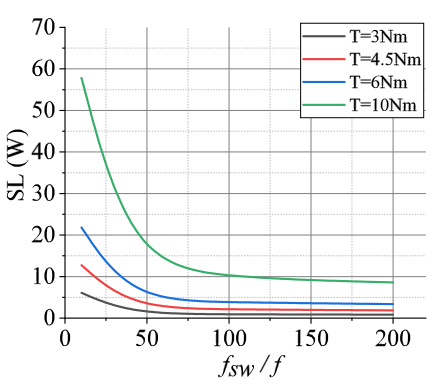

(b)

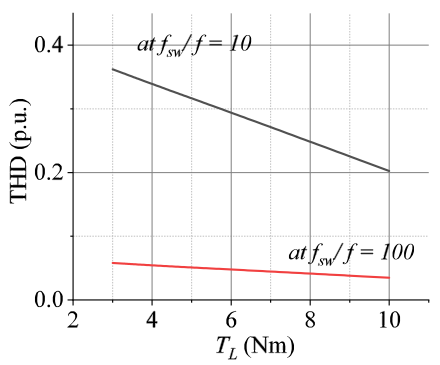

(d)

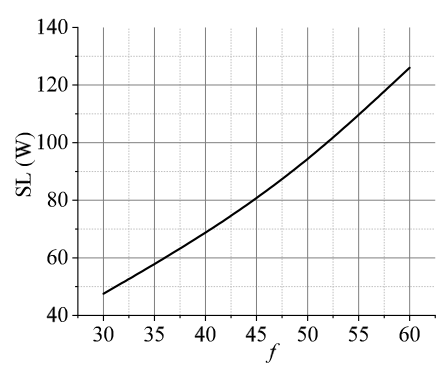

(e)

Fig. 4. Measurement results for IM-M1 (a) Variation of SL with $T_{L}^{2}$ for high and small values of $f_{S W} / f$, (b) Correlation of SL and THD variations with $f_{S W} / f$, (c) Variation of SL with $f_{S W} / f$ for different $T_{L}$, (d) Variation of THD with $f_{S W} / f$ for different $T_{L}$, (e) Impact of loading on THD at high and small $f_{S W} / f$, (f) SL variation with $f$ at constant $f_{S W} / f(=10)$ and, $T_{L}$ $(=10 \mathrm{Nm}) \cdot f$, in all these plots, $=30 \mathrm{~Hz}$.

$\left.R_{R}\left(T, f_{i}\right)\right)$ and stator-current harmonics $I_{i}$ as

$$
\begin{gathered}
P_{C u S}=3 \sum_{i=1}^{100} I_{i}{ }^{2} R_{S}\left(T, f_{i}\right) \\
P_{C u R}=3 R_{R}(T, f) I_{R 1}{ }^{2}+3 \sum_{i=2}^{100} R_{R}(T, f)\left(I_{i} \frac{X_{m i}}{X_{l R i}+X_{m i}}\right)^{2}
\end{gathered}
$$

here, $X_{m i}$ and, $X_{l R i}$ are the magnetising and rotor-leakage reactances $w . r . t$. $i$-th harmonic,

$$
P_{\text {Out }}=P_{\text {Shaft }}+P_{\text {Windage }}+P_{\text {Bearing }}
$$

and,

$$
P_{\text {Shaft }}=T_{\text {Shaft }} \omega_{r}
$$

$T_{\text {Shaft }}$ and $\omega_{r}$ are the measured values of shaft torque and rotor speed in $\mathrm{rad} / \mathrm{s}$ respectively. To reduce the measurement discrepancy, the obtained SL test data is smoothened (for correlation coefficient, $R^{2}>0.95$ ) by applying linear regression analysis with squared value of the torque. The process needs repeated measurements. The SL for the rated operating point is then calculated by multiplying the slope of the plot with 


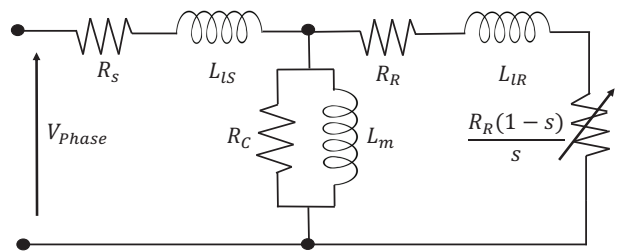

Fig. 5. Equivalent circuit of sinusoidally excited 3-phase IM. rated torque.

Fig.2 (a)-(e) show the SL values of M1 and M2 plotted against the fundamental and switching frequency for different torque values (operating points of Table II). Similarly, Fig. 6 show the measured SL values for M3 and, M4. Examination and comparison of these results for different IMs draw following observations:

- As it can be seen, the SL of the M1 is of highest value always and, lowest for M3 in all the experiments. SL values for M2 and M3 always lie between the same of M1 and M3. For example, at the rated torque at $30 \mathrm{~Hz}$ frequency with $300 \mathrm{~Hz}$ switchings, they amount to 62.18W, $57.83 \mathrm{~W}, 48.30 \mathrm{~W}$, and 52.25W for M1, M2, M3 and M4, respectively.

- SL notably increases with frequency. As Fig.2 (f) shows, the order of variation of SL with frequency is greater than one.

- The impact of carrier frequency on SL is quite prominent as expected. Low switching frequency $\left(f_{s w}\right)$ operation makes the motor current highly distorted and consequently, raise the net SL. As shown in Fig.2 (a)-(d), the impact of $f_{s w}$ shows a rapid decrement in the $f_{s w} / f$ range of $[10,50]$. For very high $f_{s w} / f$ (i.e. 100-200), SL with inverter fed IM is almost equal to that of a sinusoidally excited IM.

\section{PROPOSED SL FORMULATION}

This section presents the development of the SL model for inverter fed IM using the measured values of SL (Section III). The exact analytical formulation of the SL is not possible [22] due to non-linear behaviour of magnetic core, dependence of iron-loss coefficients on magnetisation and frequency, impact of magnetic field distribution on current density distribution within the conductors (proximity and skin effect) and, the impact of slot-tapering on flux pulsation. Development of an empirical model is, hence, the most suitable option to account quantitatively the SL. [23] presents an empirical SL model for grid-connected IMs, which includes the impact of motor geometry on SL as well. The model in [23] uses motor inductances, the number of pole pairs and, output power as input variables. However, this model is developed neglecting the impact of power supply harmonics, which limits its application to the grid connected IMs. The model will need to incorporate the following additional variables when modified to use in inverter fed IM: THD, $f_{s w}\left(\right.$ or $\left.f_{s w} / f\right)$.

\section{A. SL Model structure}

In case of inverter fed IM, the variation in SL is associated with the changes in three power supply parameters: 1.) Output power $\left(P_{\text {out }}\right)$, 2.) Supply frequency $(f)$ and, 3.) $f / f_{s w}$ ratio $(\tau)$ or current THD in motor current.

Incremental change in SL,corresponding to the changes in above three parameters is:

$$
d S L=\frac{\partial S L}{\partial P_{\text {Out }}} d P_{\text {Out }}+\frac{\partial S L}{\partial f} d f+\frac{\partial S L}{\partial \tau} d \tau
$$

Since, the three variables $\left(P_{\text {out }}\right), f$ and,$\left.\tau\right)$ are mutually exclusive, the variation (22) can be expressed as:

$$
S L=f_{1}\left(P_{\text {Out }}\right) f_{2}(f) f_{3}(\tau)
$$

Here, variation of SL with $P_{\text {Out }}$ (i.e. $f_{1}\left(P_{\text {out }}\right)$ in $(22)$ ) is recognised as reported in [23] i.e.,

$$
f_{1}\left(P_{\text {Out }}\right)=K_{S L} P_{\text {Out }}=\left(\frac{1}{p} \frac{L_{l S}}{L_{m}}\right)^{m}\left(\frac{1}{p} \frac{L_{l R}}{L_{m}}\right)^{n} P_{\text {Out }}
$$

where, $p$ is number of pole-pairs and, $L_{m}, L_{l S}, L_{l R}$ are the magnetising inductance, stator leakage inductance and rotor leakage inductance, respectively (as shown in Fig.5).

Formulation of $f_{2}(f)$ and, $f_{1}(\tau)$ uses following measurement observations (Section III):

1) Impact of frequency (for $f_{2}(f)$ ): As shown in Fig. 2 (f), $\mathrm{SL}$ increases with the fundamental frequency for constant $f_{s w}$ and, load torque $\left(T_{L}\right)$. With two trend related observations that, the rate of increment of SL with $f$ is higher than one and, SL is zero at zero $\mathrm{f}$, the simplest form of is given as:

$$
f_{2}(f)=\alpha\left(\frac{f}{f_{r}}\right)^{\beta}
$$

where, $\alpha$ and $\beta$ are the constants and $f_{r}$ is rated frequency i.e. supply frequency for rated speed, rated torque.

2) Impact of THD in motor-current (for $\left.f_{3}(\tau)\right)$ : Fig.2(a)-(b) show the measured values of current THD and SL for different switching frequency-ratio. Here it is evident that SL decreases monotonously with increment in $f_{s w} / f$ ratio following the grid SL value asymptotically. Also,the rate of change of SL with $f_{s w} / f$ depends upon the $f_{s w} / f$. This rate decreases as the ratio $f_{s w} / f$ increases i.e. the impact of switching frequency variation is high at low switching frequency. Hence, the fractional change in SL (due to change in $\tau$ only) shows a decreasing trend with $\tau$ i.e.,

$$
\frac{d f_{3}(\tau)}{f_{3}(\tau)}=\frac{d \tau}{(a+b \tau)}
$$

Solving (26) provides the general solution as:

$$
f_{3}(\tau)=\frac{k}{(a+b \tau)^{r}}+C
$$

where, $a, b, r, k$ and, $C$ are the equation constants. The expression (27) relates SL (analogously the current THD) variation with $f / f_{s w}$ for constant $T_{L}$. However, the current THD decreases as the $T_{L}$ is increased (Fig. 2(e)). This is because only the fundamental current increases when $T_{L}$ is increased. The rotor resistance to slip ratio for higher order harmonic currents observe negligible change due to higher value of their synchronous speed and thus, the change in harmonic current 
with $T_{L}$ (or slip) is very small. To incorporate this, $f_{3}(\tau)$ is further multiplied with a factor $\left(I / I_{0}\right)^{2}$. Here, $I_{0}$ is the no-load current at the same $f_{s w}$. The final expression of SL with the $f_{2}(f)$ and $f_{3}(\tau)$ is

$$
S L=\underbrace{\left(\left(\frac{1}{p} \frac{L_{l S}}{L_{m}}\right)^{m}\left(\frac{1}{p} \frac{L_{l R}}{L_{m}}\right)^{n} P_{\text {Out }}\right)}_{f_{1}\left(P_{\text {Out }}\right)} \underbrace{\left(\alpha\left(\frac{f}{f_{r}}\right)^{\beta}\right)}_{f_{2}(f)} \underbrace{\left(\frac{I}{I_{0}}\right)^{2}\left(C+\frac{k}{(a+b \tau)^{r}}\right)}_{f_{3}(\tau)}
$$

The expression (28)is summarised as

$$
S L=K_{\text {Grid }} K_{\text {Inv }} P_{\text {Out }}
$$

where,

$$
K_{\text {Grid }}=\left(\left(\frac{1}{p} \frac{L_{l S}}{L_{m}}\right)^{m}\left(\frac{1}{p} \frac{L_{l R}}{L_{m}}\right)^{n}\right)
$$

and,

$$
K_{\text {Inv }}=\left(\alpha\left(\frac{f}{f_{r}}\right)^{\beta}\right)\left(\frac{I}{I_{0}}\right)^{2}\left(C+\frac{k}{(a+b \tau)^{r}}\right)
$$

\section{B. Parameter identification}

Extraction of parameters of the SL model (28) uses both the end-conditions $\left(f=f_{r} \& \tau=0\right)$ and, non-linear regression. At first, $\alpha$ is determined as $f_{2}\left(f_{r}\right)=1 \Rightarrow \alpha=1$. Similarly, at $\tau=\infty, f_{3}(\tau)=1$ which implies, $C=1$. For identification of remaining constants, non-linear regression with the least leastsquares technique is applied and, the best empirical fit for $K_{I n v}$ is exhibited by the relation:

$$
S L=\underbrace{\left(\left(\frac{1}{p} \frac{L_{l S}}{L_{m}}\right)^{m}\left(\frac{1}{p} \frac{L_{l R}}{L_{m}}\right)^{n} P_{\text {Out }}\right)}_{f_{1}\left(P_{\text {Out }}\right)} \underbrace{\left(\left(\frac{f}{f_{r}}\right)^{2}\right)}_{f_{2}(f)} \underbrace{\left(\frac{I}{I_{0}}\right)^{2}\left(1+\frac{1}{(1+0.5 \tau)}\right)}_{f_{3}(\tau)}
$$

The obtained value of the coefficient of correlation $\left(R^{2}\right)$ with (31) is 0.98 . The values of the exponents $m$ and, $n$ are 0.95 and 0.34 , respectively.

\section{Statistical evaluation}

The errors spectrum with the proposed model for results is given in Table IV. There is no reference model in available in the literature to compare with (in terms of relative error), and, hence, only the absolute error with the measurements is compared. The model parameters are trained using the test data of M1 and M2 and, in further verification of these parameters, the SL for M3 and M4 is calculated. Comparison of the calculated and measured SL for M3 and M4 for the two extremes of $f_{S W} / f$ and $T_{L}$ is shown in Fig. (6).

Other important parameters of error-statistics wrt the proposed SL relation are given in Table V.

Noteworthy observations, derived from the Table IV and Fig. 6, regarding the prediction capability of the proposed SL model are mentioned below as:

- The proposed method ensures the accuracy of SL estimation with the estimation error less than $30 \%$ for $53.27 \%$

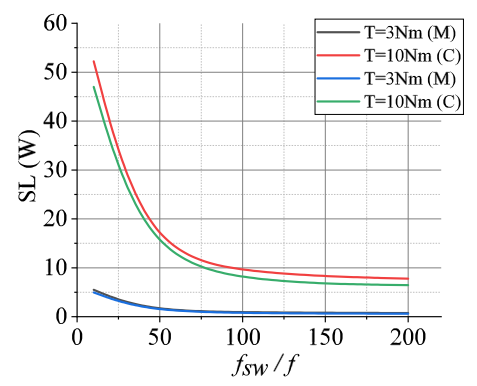

(a)

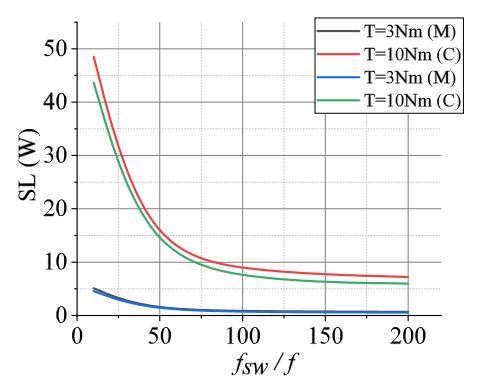

(b)

Fig. 6. Measured (M) and calculated (C) SL values for (a) M-3, (b) M-4. TABLE IV

ERror Spectrum Of the Proposed SL MOdel for Four IMS

\begin{tabular}{ccc}
\hline \hline Error(\%) & No. of test-points & \% of test-points \\
\hline $10-20$ & 44 & 17.19 \\
$21-30$ & 118 & 46.09 \\
$31-50$ & 52 & 20.31 \\
$50-70$ & 30 & 11.72 \\
$70-100$ & 10 & 3.91 \\
$i 100$ & 0 & 0 \\
\hline \hline
\end{tabular}

operating points of the test sample. Test points with error higher than $50 \%$ are $16.41 \%$ of the test domain.

- Measured SL is maximum for M1 and minimum for M3,with respective SL values equal to $1.44 \%$ and $1.03 \%$ of the rated output power. Hence, the slot geometry plays a significant role in SL deviation.

\section{CONCLUSION}

In this work, a fast and easy-to-apply empirical model is presented to estimate the SL in inverter fed IM. Experimental data of a wide range of inverter power supply parameters (i.e., $f$ and $f_{S W} / f$ ) and motors' loading $\left(T_{L}\right)$ of two IMs is analysed to develop this model. Total 128 IMs' operating points are tested in this dimensional space of $f, f_{S W} / f, T_{L}$. The model uses IMs equivalent circuit parameters, nameplate details, and directly measurable electrical quantities to estimate the SL, making the model applicable for both design and operation stages. To check the accuracy of the proposed model, two IMs (of similar rated characteristics but different geometries) are tested for SL for another 128 operating points. Comparing the calculated SL with the measured SL shows the $50 \%$ interval of the accuracy to have $83.89 \%$ of the total 256 operating points. Also, the R2 with the proposed model is estimated to be 0.997 . 


\section{REFERENCES}

[1] S. Mallik, K. Mallik, A. Barman, D. Maiti, S. K. Biswas, N. K. Deb, and S. Basu, "Efficiency and cost optimized design of an induction motor using genetic algorithm," IEEE Transactions on Industrial Electronics, vol. 64, no. 12, pp. 9854-9863, 2017.

[2] C. U. B. Paul Waide, "Energy-efficiency policy opportunities for electric motor-driven systems," Energy Efficiency Series by International Energy Agency, no. 2, pp. 1-132, 2011.

[3] H. Domeki, Y. Ishihara, C. Kaido, Y. Kawase, S. Kitamura, T. Shimomura, N. Takahashi, T. Yamada, and K. Yamazaki, "Investigation of benchmark model for estimating iron loss in rotating machine," IEEE Transactions on Magnetics, vol. 40, no. 2, pp. 794-797, 2004.

[4] Z. Gmyrek, A. Boglietti, and A. Cavagnino, "Estimation of iron losses in induction motors: Calculation method, results, and analysis," IEEE Transactions on Industrial Electronics, vol. 57, no. 1, pp. 161-171, 2010.

[5] N. Glew, "Stray load losses in induction motors: a challenge to academia," Power Engineering Journal, vol. 12, no. 1, pp. 27-32, Feb 1998.

[6] B.Heller and V.Hamata, "Harmonic field effects in induction machines," 1977.

[7] I. Boldea and S. A. Nasar, "Induction machines design handbook," vol. 31, no. 3, 2010 .

[8] A. Bonnett, "Analysis of the impact of pulse-width modulated inverter voltage waveforms on ac induction motors," IEEE Transactions on Industry Applications, vol. 32, no. 2, pp. 386-392, 1996.

[9] G. B. Reddy, G. Poddar, K. B. Nagrale, and B. P. Muni, "Synchronous pwm technique based on reference angle for induction motor traction drive application," in 2020 IEEE International Conference on Power Electronics, Drives and Energy Systems (PEDES), 2020, pp. 1-6.

[10] C.-Y. Huang, C.-P. Wei, J.-T. Yu, and Y.-J. Hu, "Torque and current control of induction motor drives for inverter switching frequency reduction," IEEE Transactions on Industrial Electronics, vol. 52, no. 5, pp. 1364-1371, 2005.

[11] I.Boldea and S. Nasar, New York: Gordon and Beach, 1970.

[12] P. Pillay, M. Al-Badri, P. Angers, and C. Desai, "A new stray-load loss formula for small and medium-sized induction motors," IEEE Trans. Energy Convers., vol. 31, no. 3, pp. 1221-1227, Sep. 2016.

[13] K. Yamazaki, A. Suzuki, M. Ohto, T. Takakura, and S. Nakagawa, "Equivalent circuit modeling of induction motors considering stray load loss and harmonic torques using finite element method," IEEE Trans. Magn., vol. 47, no. 5, pp. 986-989, May 2011.

[14] J. Wang, M. Cheng, H. Wen, M. Tong, G. Zhang, Y. Hu, and B. Chen, "Stray load loss calculation for induction motor by combination of general airgap field modulation theory and 2d fea," IEEE Transactions on Energy Conversion, pp. 1-1, 2021.

[15] H. Zhao, C. Ragusa, O. de la BarriÃ̈re, M. Khan, C. Appino, and F. Fiorillo, "Magnetic loss versus frequency in non-oriented steel sheets and its prediction: Minor loops, pwm, and the limits of the analytical approach," IEEE Transactions on Magnetics, vol. 53, no. 11, pp. 1-4, 2017.

[16] E. B. Agamloh, "An evaluation of induction machine stray load loss from collated test results," IEEE Transactions on Industry Applications, vol. 46, no. 6, pp. 2311-2318, Nov 2010.

[17] M. Basic, D. Vukadinovic, and M. Polic, "Stray load and iron losses in small induction machines under variable operating frequency and flux: A simple estimation method," IEEE Transactions on Energy Conversion, vol. 33, no. 2, pp. 869-876, June 2018.

[18] M. Aoulkadi and A. Binder, "Comparison of different evaluation methods to determine stray load losses in induction machines with eh-star method," in 2007 IEEE Int. Electric Machines Drives Conf., vol. 1, May 2007, pp. 519-524.

[19] M. Al-Badri, P. Pillay, and P. Angers, "Induction machine rapid performance test," IEEE Transactions on Industry Applications, vol. 55, no. 5, pp. 4685-4691, 2019.

[20] Q. Zhu, Q. Wu, W. Li, M.-T. Pham, and L. Zhu, "A general and accurate iron loss calculation method considering harmonics based on loss surface hysteresis model and finite-element method," IEEE Transactions on Industry Applications, vol. 57, no. 1, pp. 374-381, 2021.

[21] J. Cheaytani, A. Benabou, A. Tounzi, and M. Dessoude, "Stray load losses analysis of cage induction motor using 3-d finite-element method with external circuit coupling," IEEE Trans. Magn., vol. 53, no. 6, pp. 1-4, June 2017.

[22] A. Boglietti, A. Cavagnino, L. Ferraris, and M. Lazzari, "Induction motor equivalent circuit including the stray load losses in the machine power balance," IEEE Trans. Energy Convers., vol. 23, no. 3, pp. 796803, Sep. 2008.

[23] R. Kumar, P. Kumar, T. Kanekawa, and K. Oishi, "Stray loss model for induction motors with using equivalent circuit parameters," IEEE Transactions on Energy Conversion, vol. 35, no. 2, pp. 1036-1045, 2020. 